\title{
System Level Design Trade-Offs with the SKA Costing Tool
}

\author{
R.C. Bolton ${ }^{1}$, P. Alexander ${ }^{1}$, D.C. Ford ${ }^{1}$, T. Colegate $^{2}$, P. Hall ${ }^{2}$ \\ ${ }^{1}$ Cavendish Astrophysics Group, Cavendish Laboratory, University of Cambridge \\ 2 International Centre for Radio Astronomy Research / Curtin University, Perth, Australia
}

\begin{abstract}
Within the SKADS programme we have developed SKACost, the costing and design tool to be more flexible (see Ford et al. 2009, 2010, these proceedings). The original version of SKACost was presented in Chippendale et al. (2007), whilst the original SKADS costing was a spreadsheet based approach (see Alexander et al. 2007). The new version of SKACost combines the best of both approaches, incorporating the complexity of a full telescope design with the ease of use of a Graphical User Interface. Telescope designs are broken down into logical hierarchical structures enabling experts to study their design areas in detail whilst system designers can take a more global approach. Here we briefly present the model used to populate this costing tool and show how it can be used to study system level scaling relations.
\end{abstract}

\section{Introduction}

In this paper we show how the SKA Costing tool is populated with hierarchical telescope designs and give some important examples that show how useful such a tool can be when studying the impact that varying certain parameters can have on the overall cost of a telescope or its sub-systems.

A full description of the tool itself has been presented by Ford et al. (2009), here we concentrate on how the tool can be used.

\section{The SKA Costing tool}

The concept of "Design Blocks" was introduced in SKADS for D\&C 1 (see SKA Memo 93, Alexander et al. 2007). Within the SKADS system level design work, we considered hierarchical telescope designs made up of discrete logical blocks and components. This initial work was implemented in a spreadsheet, which, owing to its complexity, was difficult to navigate for all but those few users very familiar with it. Separately to this and also following a hierarchical structure, the original version of the SKA costing tool, SKACost, was developed by the then ISPO, with work led by Peter Hall, Aaron Chippendale, John O'Sullivan and Tim Colegate (see SKA Memo 92, Chippendale et al. 2007).

We have updated the costing tool to generate a new version of SKACost which combines the best of these approaches including advanced cost modelling and uncertainty handling (using Monte Carlo techniques to estimate overall cost uncertainties), the ability to swap telescope designs with relative ease and with a GUI for easy browsing and editing. This allows users with a variety of levels of expertise to access the tool and study the system aspects relevant to their work - for example, component level items can be created and studied by the relevant engineering experts whilst system designers can make sweeping changes to the telescope design without the need to worry about the fine detail. The hierarchical structure ensures that when the "big things" are changed, the "little things" will follow.
Figure 1 (on page 158) shows the full GUI page of the tool, with a telescope design in it called "SKADS AA and Dishes SKA", which represents the SKADS vision for the SKA, as presented in SKA Memo 111 (Bolton et al. 2009). The main GUI window gives information on the hierarchical structure of the telescope and, on the right hand side, details of the cost and power estimates accrued for the highlighted design blocks. By clicking on the green arrows or on the hierarchy diagram users can navigate through the design, "drilling down" to the appropriate level.

The top level of the SKA is made up of eight main blocks in this design, these are: (1) "SKADS SKA Infrastructure", containing some roads, buildings and trenching for data links; (2) "Dishes Outer", representing the 1500 dishes that are spread along spiral arms, and the data links required to bring their data to the correlator; (3) "Dishes Core", representing the 1500 dishes in the core, and their data links; (4) "AAhi Core" the 165 aperture array stations in the core, functioning in the $300 \mathrm{MHz}$ to $1 \mathrm{GHz}$ band and their data links; (5) "SKADS AAhí Outer", the outer 85 AAhi Stations and their data links; (6) "AAlo Core", the low frequency aperture array collectors in the core, operating from $70 \mathrm{MHz}$ to $450 \mathrm{MHz}$, and the data links for these; (7) "SKADS AAlo Outer", the outer AAlo stations - which are assumed to share data links with the AAhi outer stations; and (8) a block called "Correlator" which models the correlator cost for the dishes and aperture arrays as two separate correlators.

Figure 2 shows the hierarchy diagram centered on the SKADS AAhi Outer block. This shows that this block has a "parent" (the SKA block) and two direct children (the AAhi Stations and the data links for these stations). These in turn are made up of several sub-design blocks, representing subsystems in the design. For example, the AAhi Station block contains a sub-block representing the infrastructure associated with each station. This is shown in figure 3 . The infrastructure design block contains several components including steel supports to make the frame that will hold the antennas (these are ordered by unit length) and tarpaulin to cover the station to 


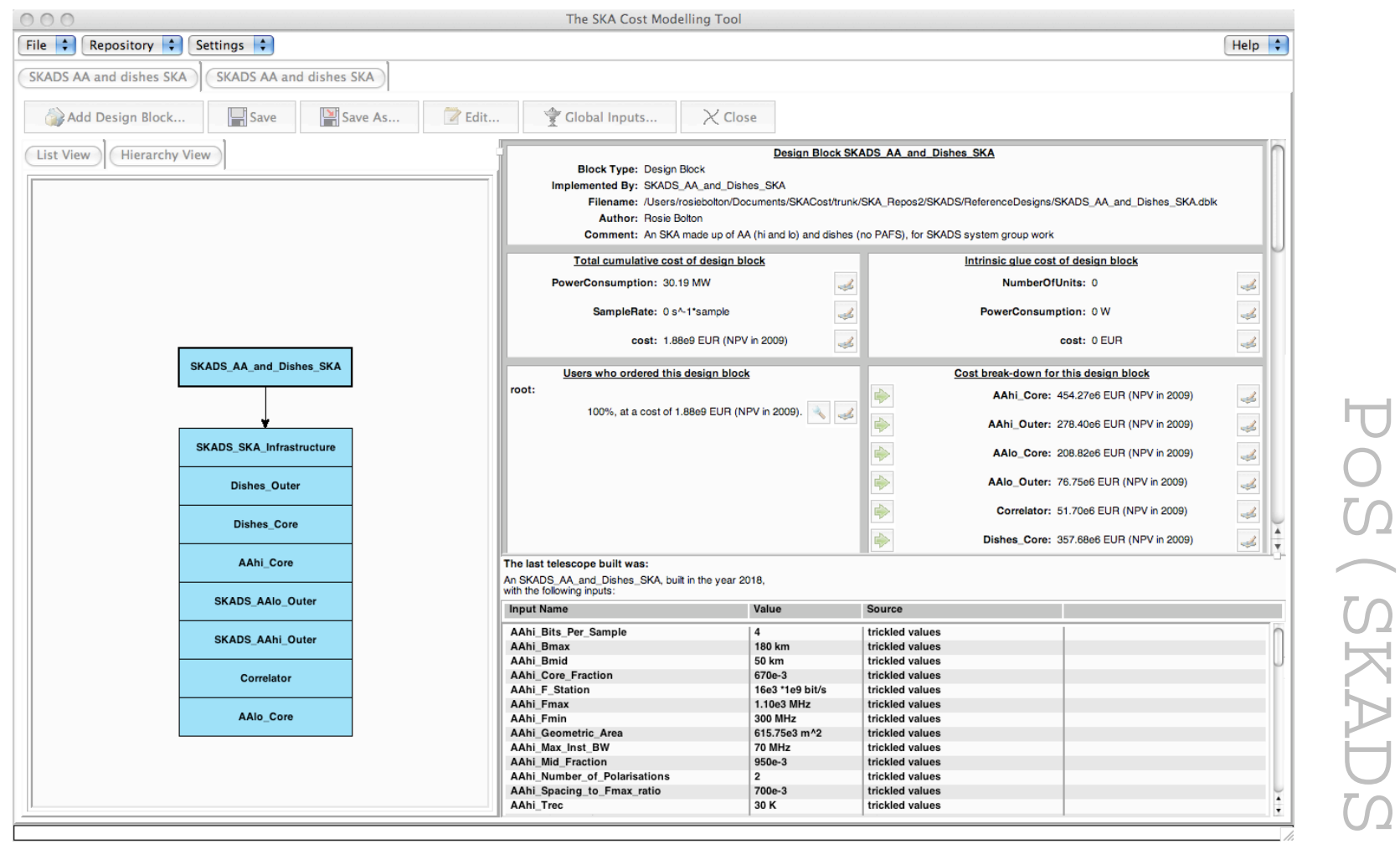

Fig. 1: Screenshot of the GUI.

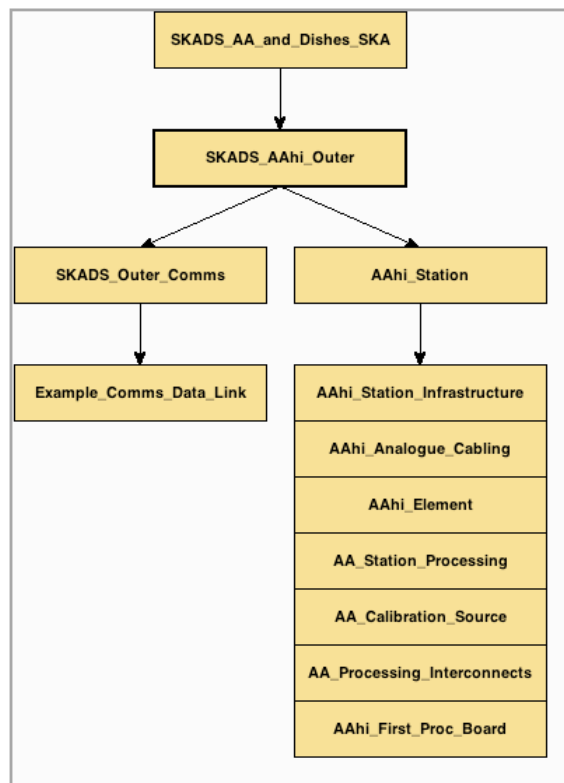

Fig. 2: The hierarchy diagram for the outer AAhi stations.

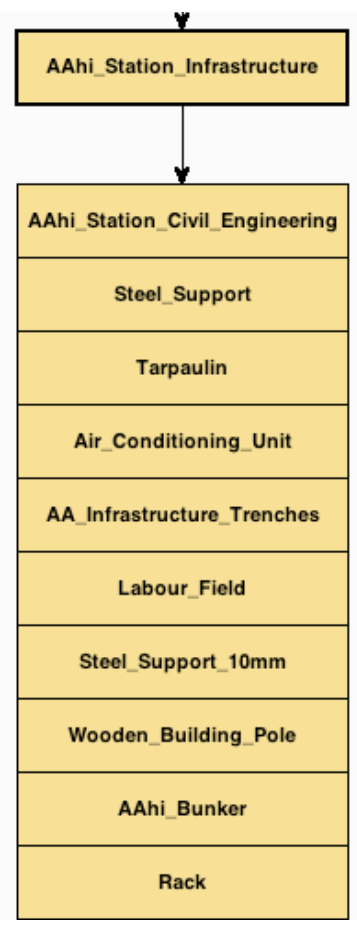

Fig. 3: Hierarchy diagram for the AAhi station infrastructure, showing its children.

provide protection from the weather, which is ordered by unit area. 


\begin{tabular}{|c|c|}
\hline \multicolumn{2}{|c|}{$\begin{array}{l}\text { The last telescope built was: } \\
\text { An SKADS_AA and_Dishes_SKA, built in the year 2018, } \\
\text { with the following inputs: }\end{array}$} \\
\hline Input Name & Value \\
\hline AAhi_Bits_Per_Sample & 4 \\
\hline AAhi_Bmax & $180 \mathrm{~km}$ \\
\hline AAhi_Bmid & $50 \mathrm{~km}$ \\
\hline AAhi_Core_Fraction & $670 \mathrm{e}-3$ \\
\hline AAhi_F_Station & $16 \mathrm{e} 3 * 1 \mathrm{e} 9 \mathrm{bit} / \mathrm{s}$ \\
\hline AAhi_Fmax & $1.10 \mathrm{e} 3 \mathrm{MHz}$ \\
\hline AAhi_Fmin & $300 \mathrm{MHz}$ \\
\hline AAhi_Geometric_Area & $615.75 \mathrm{e} 3 \mathrm{~m}^{\wedge} 2$ \\
\hline AAhi_Max_Inst_BW & $70 \mathrm{MHz}$ \\
\hline AAhi_Mid_Fraction & $950 \mathrm{e}-3$ \\
\hline AAhi_Number_of_Polarisations & 2 \\
\hline AAhi_Spacing_to_Fmax_ratio & $700 \mathrm{e}-3$ \\
\hline AAhi_Trec & $30 \mathrm{~K}$ \\
\hline AAlo_Bits_Per_Sample & 4 \\
\hline AAlo_Bmax & $180 \mathrm{~km}$ \\
\hline AAlo_Bmid & $50 \mathrm{~km}$ \\
\hline AAlo_Core_Fraction & $670 \mathrm{e}-3$ \\
\hline AAlo_F_Station & $16 \mathrm{e} 3 * 1 \mathrm{e} 9 \mathrm{bit} / \mathrm{s}$ \\
\hline AAlo_Filling_Factor & $950 \mathrm{e}-3$ \\
\hline AAlo_Fmax & $450 \mathrm{MHz}$ \\
\hline AAlo_Fmin & $70 \mathrm{MHz}$ \\
\hline AAlo_Geometric_Area & $6.36 \mathrm{e} 6 \mathrm{~m}^{\wedge} 2$ \\
\hline AAlo_Max_Inst_BW & $-1 \mathrm{MHz}$ \\
\hline AAlo_Mid_Fraction & $950 \mathrm{e}-3$ \\
\hline AAlo_Number_of_Polarisations & 2 \\
\hline AAlo_Nyq_freq_to_Fmax_ratio & $2220-3$ \\
\hline
\end{tabular}

Fig. 4: Example of some of the parameters used to build a particular telescope design instance.

\section{Using parameterised designs to study trade-offs}

Telescope designs are parameterised by their top level inputs (examples of which are shown in figure 4). This is important because it enables users to easily investigate the effect of changing a parameter. Changing one of these input parameters does not only change the cost, the cost changes because it forces a different telescope to be built users can see what has changed. For example, halving the total collecting area required for the dishes would mean that rather than 3000, only 1500 dishes were built.

SKACost is very good at revealing how certain parameters affect costs because it can be used to run "parameter surveys" where a single input for a design block (at any level in the hierarchy) is varied over a range of values and the resulting build cost of that design block is recorded.

One example of how these surveys can produce interesting results concerns the design of the AAlo stations. A schematic diagram of an AAlo station is shown in 5. The (analogue) data from the AAlo antennas are taken, via copper cables, to small shielded processing "boxes" (the purple rectangles in figure 5) where they are digitised and a first stage of beamforming is carried out.

For a station of fixed size, with a fixed number of antennas we can investigate the impact that changing the number of these processing boxes has on the total cost of a station. More boxes mean that the cables from the antennas are shorter, saving money, but more boxes must be bought. Additionally, there must be an integer number of the beamforming processing boards in each box, so varying the number of signals brought into each box alters the efficiency with which these beamformers are used - a "bad" choice of box number means that more processing boards are required.

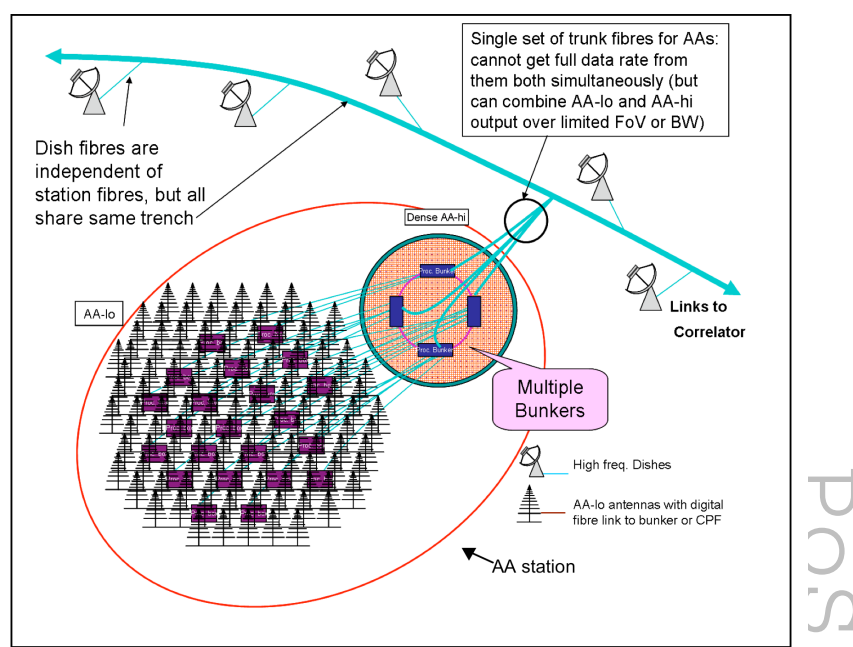

Fig. 5: Schematic diagram of an AA station, where AAlo and AAhi antennas are placed in adjacent circular stations.

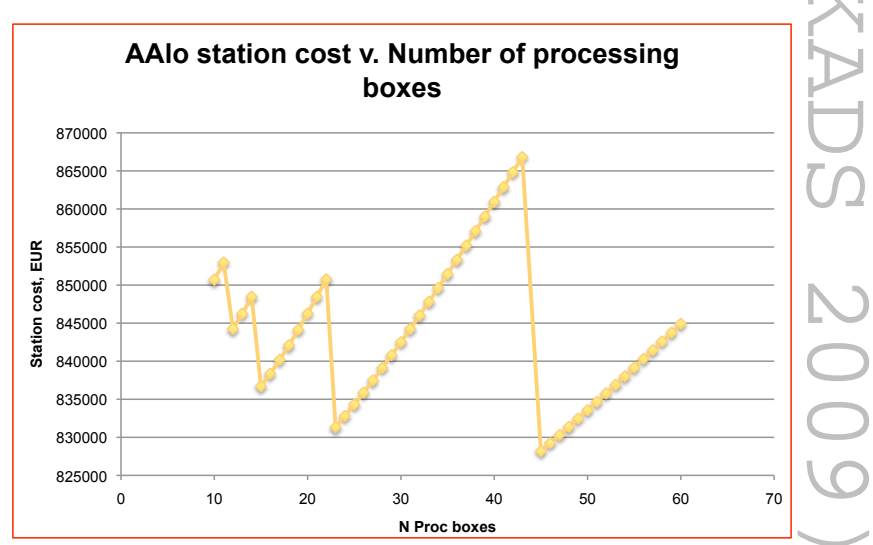

Fig. 6: The data output from the costing tool when tracing how the cost of an AAlo station is affected by the number of processing boxes used.

We can also use SKACost to show us how the cost of a data link, carrying a fixed data rate, varies with the length of the link. The results are shown for a 16Tbit/s link in figure 7 . The underlying slope in the stepped curve is due to the smoothly increasing length of cable required. Steps in the cost represent technology break points, which are built into the design blocks - for example, if the link is short enough, the tool will use the "cheap" short range lasers, only opting to use better lasers (which are more expensive) for links beyond the break point. In this way, the tool always selects the most appropriate option for a link of a given data rate and length. The logic that produces the desired behaviour is encoded within a snippet of Python code that resides in the desgin block file.

The clear and strong jumps in this graph have led us to consider the impact that the broad distribution would have on the cost of all the AA links. In the costing tool we parameterised the AA distribution with five numbers: the total number of stations, the data rate per station, the fraction of these stations within a $2.5 \mathrm{~km}$ radius core, the maximum distance the AA sta- 


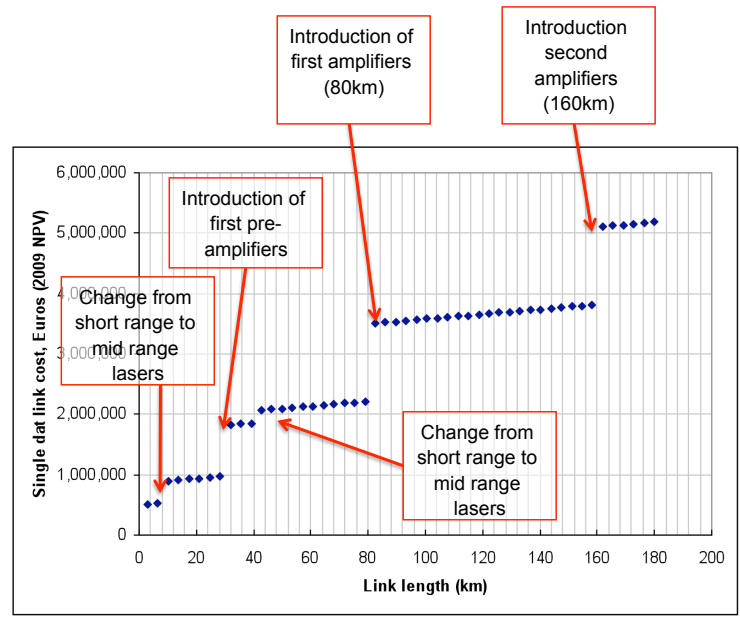

Fig. 7: Cost curve for a data link carying 16Tbits/s of data. The link length was varied in the costing tool.

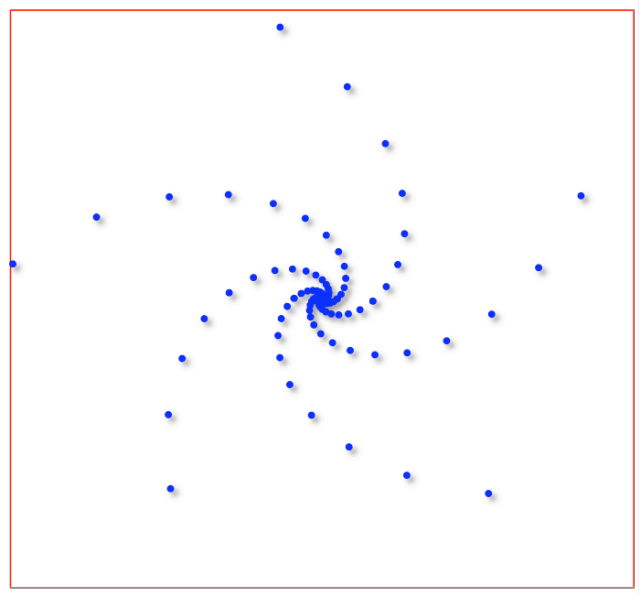

Fig. 8: A possible 250 AA station layout with $67 \%$ of the collectors in the core and the remaining 85 stations spaced at equal logarithmic intervals along 5 spiral arms out of $180 \mathrm{~km}$ radius. $95 \%$ of all the collectors lie within $90 \mathrm{~km}$ of the core.

tions go out to (BMax) and one extra parameter, BMid. BMid is the distance from the centre within which $95 \%$ of the collectors are placed. By assuming a logarithmic distribution between $2.5 \mathrm{~km}$ and $\mathrm{BMid}$ and a second logarithmic distribution between BMid and BMax, the distance to each station is defined.

Examples of two such distributions are shown in figures 8 and 9. The first shows a configuration where BMid has been chosen so that the ratio between station distances before and after BMid is the same (i.e. there is just one logarithmic distribution - the value of BMid is $87 \mathrm{~km}$ ). The second configuration shows an extreme example where BMid has been set to just $10 \mathrm{~km}$. Both layouts have a maximum extent (BMax) of $180 \mathrm{~km}$ from the centre.

Because we have parameterised the description of the layout, we can use the costing tool to show us how the total cost of the AA data transport for the outer stations varies with the BMid parameter. The results are shown in figure 10 .
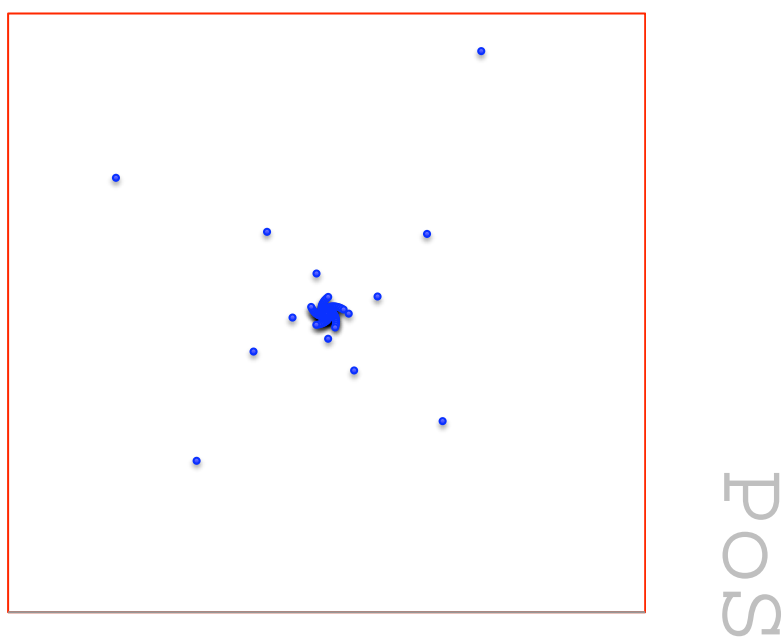

Fig. 9: A possible alternative 250 AA station layout, the same as in figure 8 but with $95 \%$ of the collectors within $10 \mathrm{~km}$ of the centre and with the remainder logarithmically spaced on spiral arms out to $180 \mathrm{~km}$ radius.

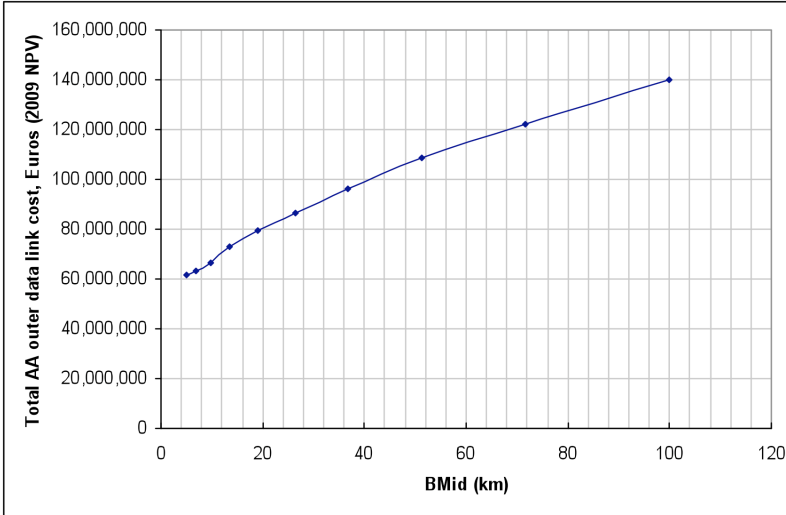

Fig. 10: Total AA data links cost as a function of BMid, the radius within which $95 \%$ of the stations are placed.

The difference between the costs of the two configurations shown here is about $€ 70$ million which is a significant potential saving and amounts to $5 \%$ of the total SKA budget. Of course, the uv-coverage of a condensed AA station layout will be very different to the un-condensed version, so these different layouts must be tested against the science cases before we can decide whether the cheaper one is acceptable. Results from some simulations done in SKADS are anticipated very shortly.

\section{Summary}

SKACost, the SKA cost/performance tool has been substantially developed in SKADS, with input from an international team. The SKADS vision for the SKA with dishes and aperture arrays has been implemented in a scalable fashion in the tool, and SKACost has been used to inform design decisions within SKADS.

Telescope designs are represented by a logical hierarchical structure. Alternative telescope designs can be investigated by 
"unplugging" one design block and replacing it with another. Currently the tool provides a useful starting point for visualising the overall system and making trade-offs.

Parameterised designs allow the impact of varying any of the inputs to be assessed: the costing tool can be used to generate graphs showing these trends automatically. These trade-offs have already helped to inform the system design work within SKADS, and the tool is also the basis for the overall SKA Costing work that had taken place in the SKADS programme.

Moving forward, the tool and the designs encapsulated within will continue to be useful in the SKA design process, since the information in the design blocks naturally forms a database of the system. This database is easily extensible to include basis of estimates for costs and improved power information, which will be essential as the SKA project moves forward.

Acknowledgements. This work was supported by the European Commission Framework Program 6, Project SKADS, Square Kilometre Array Design Studies (SKADS), contract no 011938.

\section{References}

Alexander, P., et al., 2007, SKA Memo 93.

Bolton, R.C., et al., 2009, SKA Memo 111.

Chippendale, A., et al., 2007, SKA Memo 92.

Ford, D., et al., 2009, SKADS Memo T23.

Ford, D., et al., 2010, in Proc. Wide Field Science and Technology for the SKA, Limelette, Belgium, S.A. Torchinsky et al. (eds), ASTRON, ISBN 978-90805434-5-4 